Rev. Elev. Méd. vêt. Pays trop., 1968, 21, 4 (455-461).

\title{
Rickettsiose des monocytes observée chez le porc au Sénégal
}

\author{
par M. RIOCHE ef P. BOURDIN
}

\begin{abstract}
RÉSUMÉ
Une enzaotie est observée pendant la saison frâche dans un élevage porcin des environs de Dakar et rapportée à une rickettsiose. Les symptômes, l'évolution et les lésıons micro et macroscopıques sont décrits. Curativement, seule l'auréomycine est active sous réserve d'être utilisée de façon précoce. La rickettsie observée n'est pas Rıckettsia suis, mais ainsi que le montrent les expérimentałions solt Rickettsia bovis, soit une espèce voísine commune aux suidés et aux bovidés. L'agent causal n'a pu être cultivé Cette rickettsiose apparaît chez des animaux prémunis à l'occosion d'un choc physiologique.
\end{abstract}

Au cours de la saison fraîche 1965-66, une maladie porcıne est observée dans un élevage des environs de Dakar. Les deux premiers malades (porcs importés de France depuis un mois environ) sont amenés au Laboratoire. L'un des anımaux meurt pendant le transport, l'autre, quelques jours après son arrivée au Laboratoire. Chez les deux porcs, des rickettsies sont observées en grand nombre dans les monocytes du sang périphérique et des organes.

Puis les cas se succèdent de façon sporadique pendant environ trois mois. L'affection ne sévit que sur les animaux âgés de 3 à 6 mois. Les rickettsies sont observées chez les 10 malades qui ont pu faire l'objet de prélèvements.

\section{I. - ÉTUDE CLINIQUE ET ANATOMOPATHOLOGIQUE}

\section{Symptômes.}

Le premier signe est l'abattement. L'animal reste dans un coin, indifférent à ce qui l'entoure. Il ne mange pas et n'a aucune réaction quand on l'excite.

En général, la fièvre est élevée d'emblée : l'hyperthermie atteint $40^{\circ} \mathrm{C}$ et peut même dépasser $41^{\circ} \mathrm{C}$, le corps est parcouru de frissons.
On note ensuite l'apparition de plaques congestives surtout au niveau des extrémıtés, tandis que s'installent la dyspnée et une toux sèche, fréquente. La respiration devient discordante, la congestion sous-cutanée évolve vers la cyanose et l'on est frappé par la coloration violette du groin, des oreilles et de l'extrémité des membres. Il y a souvent des symptômes nerveux qui se traduisent par une parésie du train postérieur. II y a parfois entérite.

A la fin de la maladie, le décubitus est permanent et I'animal meurt en hypothermie. Sans traitement, la mort est de règle.

\section{Evolution.}

Elle est en général rapide. Elle peut être suraigue entraînant la mort en quelques heures.

Le plus souvent l'affection dure 3 à 5 jours (forme alguë) (fig. 1).

Dans un cas (fig. 2), l'évolution a duré 20 jours. Au début, la maladie apparaît sous son aspect aigu. Bien que la température soit presque normale, les symptômes pulmonaires sont alarmants ef l'animal semble épuisé. Cet état persiste 4 jours, puss l'état général s'amélıare, l'animal se relève, mange et les signes pulmonaires 


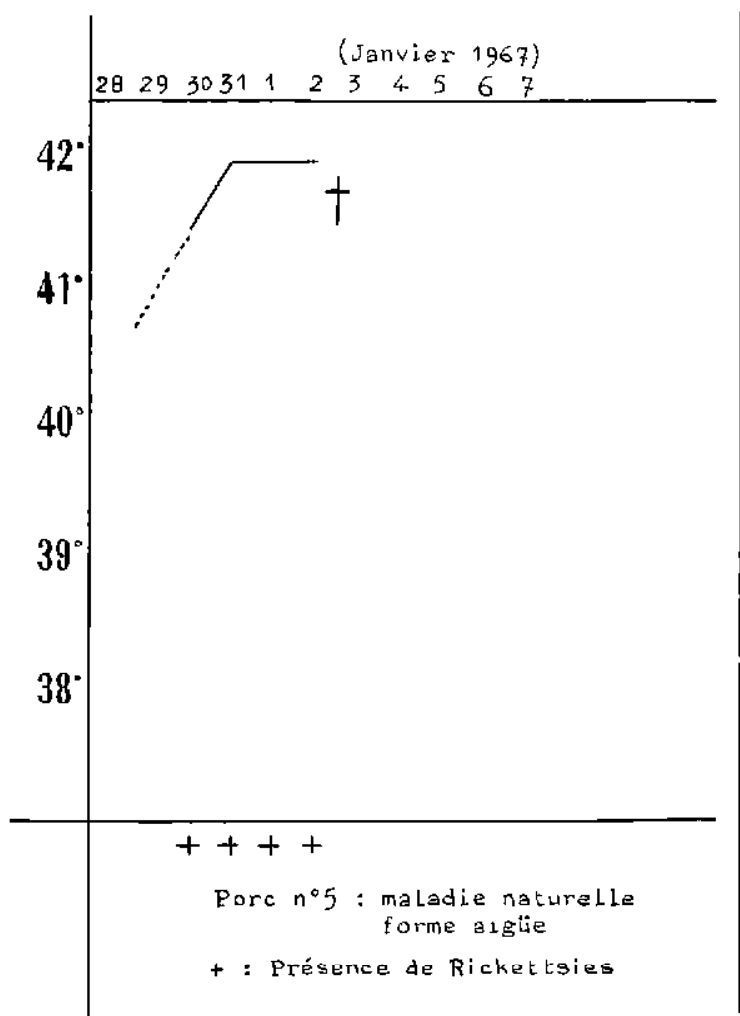

s'atténuent; la température se maintient cependant à $3902-3903$. Après 6 jours de rémission, la température monte à 3908 tandis que les symptômes réapparaissent. Le porc meurt 10 jours après cette rechute pendant lesquels la température se maintient aux environs de $40^{\circ} \mathrm{C}$ tandis que les symptômes s'aggravent.

\section{Lésions.}

\section{a) Macroscopiques.}

Le cadavre est souvent atteint de congestion généralisée.

Les épanchements séreux du péricarde, des cavités pleurales et péritonéales sont fréquents et plus ou moins abondants.

Les lésions hémorragiques sont constantes mais leur localisation est variable. Elles s'observent le plus souvent sur le coeur, à la base des artères aorte et pulmonaire et sur le rein.

Les centres nerveux sont légèrement congestionnés.

Le cœur est congestionné et atteint le plus souvent de lésions hémorragıques (pétéchies. suffusions et placards hémorragiques).

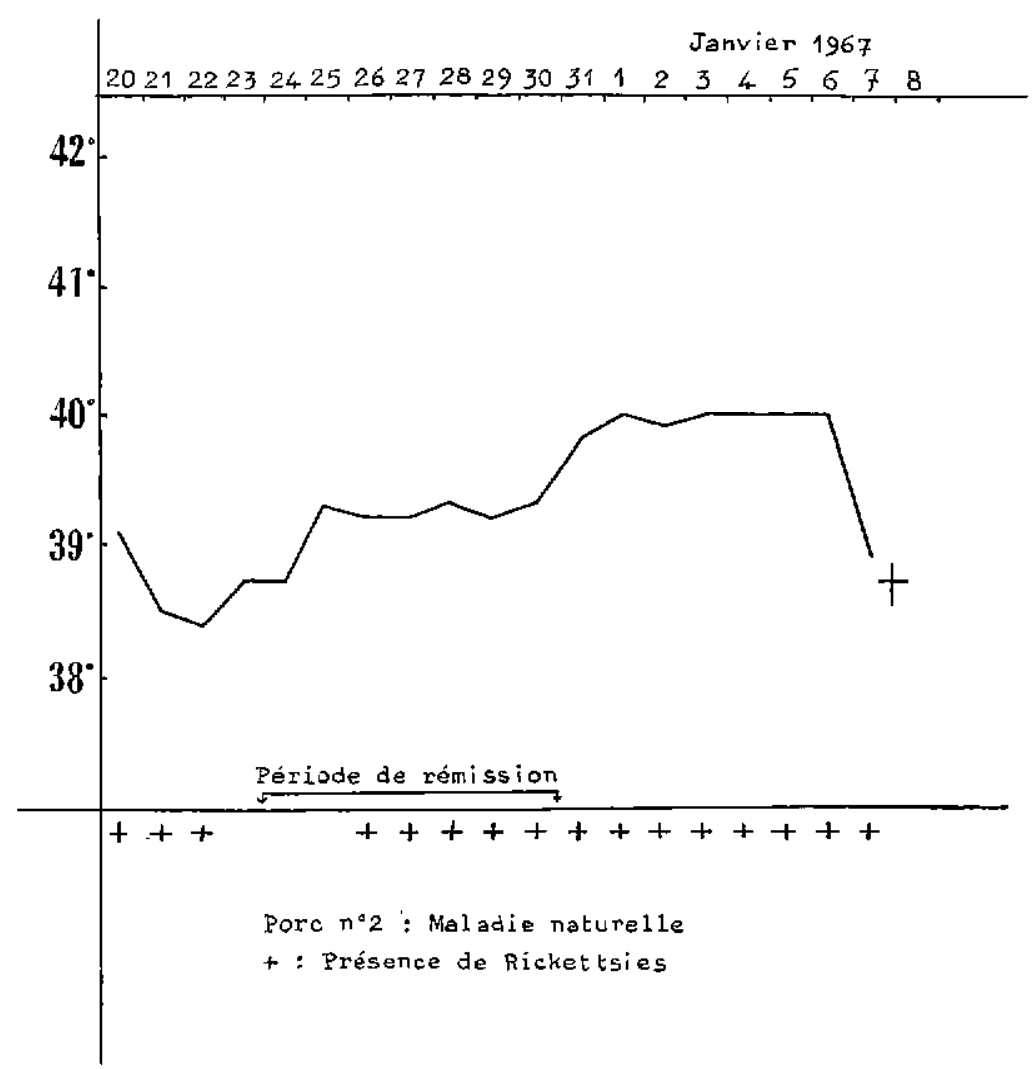


Il y a bronchopneumonie ef cedème aigu du poumon.

Le foie ef l'intestin sont congestionnés (on ne voit jamais de lésions hémorragiques au niveau de la valvule iléo-coecale).

La rate est hypertrophiée, à un degré variable.

Sur le reın, congestionné, il est fréquent d'observer des pétéchies, plus ou moins nombreuses.

Il y a parfois gastrite hémorragique.

La muqueuse vésicale peut être congestionnée.

Les ganglions thoraciques et abdominaux toujours congestionnés et succulents, sont souvent le siège d'hémorragies plus ou moins étendues.

\section{b) Microscopiques.}

Centres nerveux : pas de lésions visibles. Légère congestion de la pie mère.

Cour : congestion plus ou moins intense. Plages de nécrose où les fibrilles musculaires sont dissociées, perdent leur striation et se lysent. Hémorragles diffuses de l'épicarde.

Poumon : foyers de bronchopneumonie avec forte infiltration lymphocytaire. Epanchements fibrineux ou hémorragiques dans les alvéoles dont les parois sont épaissies.

Foie : congestion importante en particulier des capillaires intralobulaires. Les cellules hépatiques sont souvent atteintes, le cytoplasme montre une dégénérescence granuleuse accompagnée du phénomène de pycnose au niveau du noyau. II en résulte une dislocation des travées de Remak. Les lésions nucléaires sont plus nombreuses que celles du cytoplasme.

Rein : congestion intense et néphrite épithéliale atteignant tout le parenchyme. Les cellules desquament dans la lumière du tube et les lésions sont à la fois nucléaires et cytoplasmiques. Le glomérule semble indemne.

Rate : congestion des sinus. Infiltration hématique des corpuscules de Malpighi.

Ganglions lymphatiques : aspect congestif et hémorragies diffuses plus ou moins étendues.

Sang : le tableau hématologique est celui de la rickettsiose générale bovine. Les rickettsies sont observées dans les monocỳtes et, assez fréquemment dans les polynucléaires neutrophiles.

Ces lésions microscopiques sont identiques à celles de la Rickettsiose générale bovine (RIOCHE, 1967).

\section{Diagnostic.}

\section{a) De la rickeffsiose.}

II se fait par l'observation des rickettsies. Elles sont trouvées en grand nombre dan's les monocytes et assez souvent dans les polynucléaires neutrophiles. Ces microorganismes présentent les caractères morphologiques et tinctoriaux des rickettsies des monocytes et peuvent être rapportées au genre Erlichia (Moshkovskii 1945).

\section{b) Diagnostic différenfiel.}

Les tableaux clinique et nécropsique pouvant évoquer les pestes porcines africaine et classique, il était nécessaire, malgré la présence des rickettsies, d'éliminer ces deux affections. Les recherches d'entéro-virus et de bactéries pathogènes ont été aussi effectuées.

\section{b-1) Peste parcine africaine.}

Des broyats de rate de malades sont soumis au test de MALMQUIST et HAY (1960) (hémadsorption sur cultures et subcultures de leucocytes). Dans tous les cas, ce test reste négatif.

\section{b-2) Peste porcine classique.}

Deux porcelets sont inoculés dans le péritoıne, le premier avec un broyat de rate prélevée sur un malade, le second avec le même broyat préalablement inactivé avec du sérum hyperimmun contre la peste porcine (*). Ces deux animaux meurent après avoir présenté la symptomatologle décrite plus haut et les rickettsies sont observées en grand nombre dans leur sang et les frottis d'organes.

b-3) Recherche d'enterovirus.

Les selles des malades traltées à l'éther et filtrées sont inoculées à des cultures de tissus. Aucun enterovirus n'est isolé.

\section{b-4) Maladies microbiennes.}

Les ensemencements réalisés sur milieux aérobies et anaérobies à partir' de la rate, du foie, du poumon et de la moelle osseuse ne permettent pas d'isoler de bactérie pathogène.

L'élimination de ces affections permet donc d'assurer qu'il s'agit d'une rickettsiose.

$\left(^{*}\right)$ Tous les broyais d'organes inoculés étaient additıonnés de quantıtés identıques de pénıcillıne et de sireptomycine (500 Ul pénicilline et 300 gamma de streptomycine par millilitre de broyat. 


\section{Traitement.}

Seuls quelques animaux on été traités et les résultats sont décevants. La pénicilline $(1.000 .000$ d'U I intramusculaire) est inefficace.

L'auréomycine per os est inefficace.

L'auréomycine injectée par voie intrapéritonéale ou intramusculaire $(500 \mathrm{mg}$ par jour pendant 4 jours) n'a amené la guérison que si elle était administrée dès le premier jour de la maladie. Son action est illusoire dans les formes suraiguës.

\section{II. - ÉTUDE EXPÉRIMENTALE}

10 Reproduction expérimentale de la maladie.

La Rickettsiose a pu être reproduite expérlmentalement par inoculation à des porcelets de suspensions de broyats de rate ou de cerveau prélevés sur des animaux morts de la maladıe.

L'inoculum est constitué dans les deux premiers cas par le surnageant d'une suspension de rate ou de cerveau au $1 / 10^{\circ}$ dans le sérum physiologique, centrifugée pendant 10 minutes à $2.000 \mathrm{t} / \mathrm{min}$.
Dans le troisième cas, il s'agit d'un filtrat de broyat de rate : broyat au $1 / 10^{\circ}$ en sérum physiologique centrifugé pendant 20 minutes à $8.000 \mathrm{t} / \mathrm{min}$. filtré par pression sur filtre EKS (SEITZ) et additionné de 500 Ul de pénicilline et de $300 \mu \mathrm{g}$ de streptomycine par millilitre.

Les 3 porcelets utilisés sont mis en observation une semaine avant l'inoculation. Tous les jours, leur température est prise et un frottis de sang est examiné. Aucun d'eux n'est malade et la rickettsie n'esł pas observée dans les étalements de sang.

a) Inoculation de matière cérébrale.

$15 \mathrm{ml}$ de suspension sont inoculés dans le péritane d'un porcelet. L'animal tombe malade le $5^{\mathrm{e}}$ jour après l'inoculation et meurt le $16^{\mathrm{e}}$ jour après avoir présenté les symptômes déjà décrits. (Fig. 3).

\section{b) Inoculation de rate.}

$7 \mathrm{ml}$ de suspension de rate sont inoculés dans le péritoine d'un porcelet. 11 jours après, il présente les premiers symptômes et meurt après 48 heures de maladie.

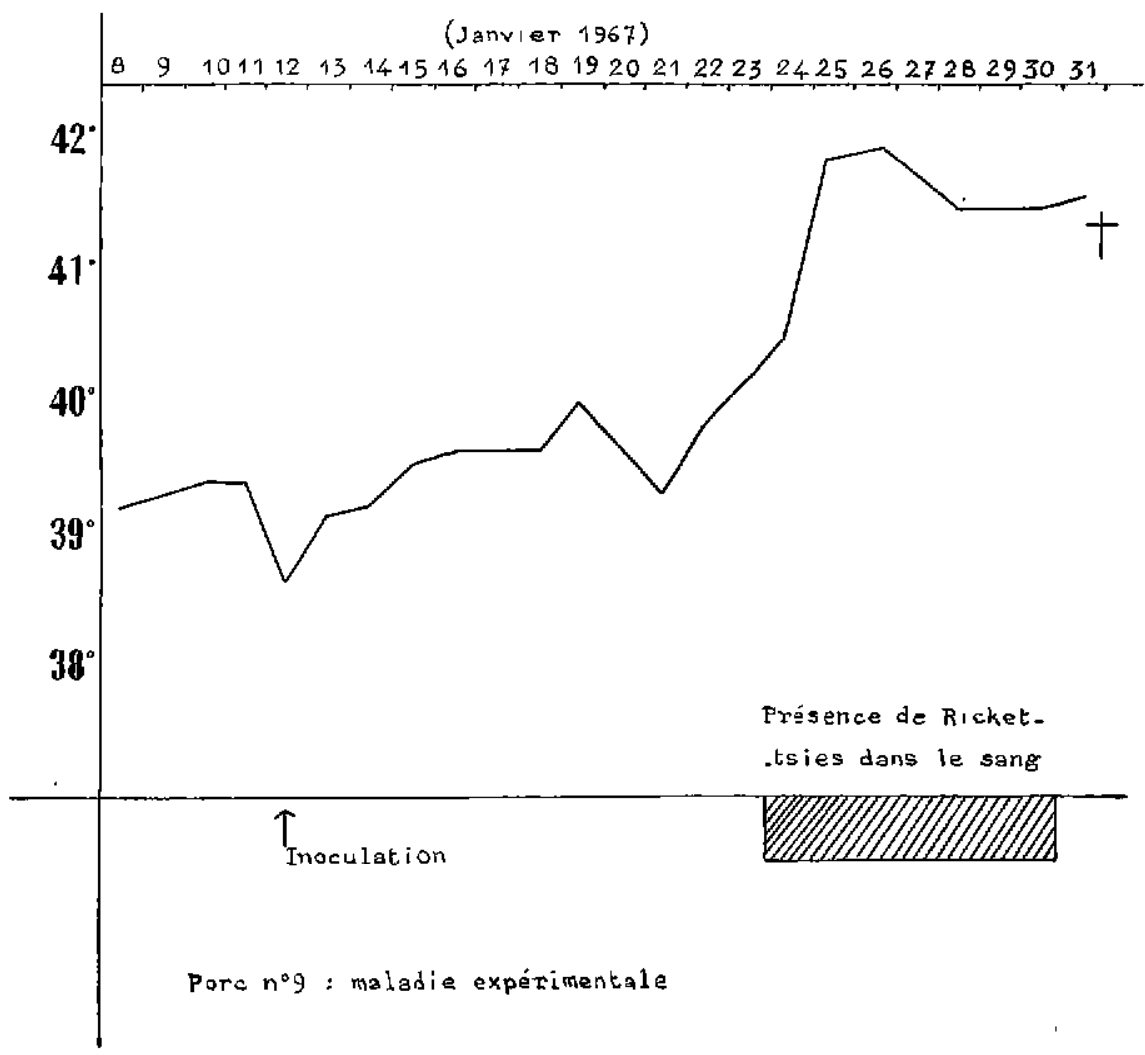




\section{c) Inoculation d'un filtraf de rate.}

Le filtrat de rate est injecté à la dose de $20 \mathrm{ml}$ dans le péritoine d'un porcelet. Trois jours après l'inoculation, l'hyperthermie s'installe et les symptômes de rickettsiose apparaissent. Cet animal meurt après 4 jours de maladie.

Chez ces trois porcelets, la rickettsie est observée régulièrement durant la maladie. Par ailleurs, les recherches d'autres agents infectieux restent négatives.

\section{Essais de transmission à la souris et d'adap-} tation à l'œuf embryonné.

\section{a) Essai de transmission à la souris.}

Les inoculations à la souris adulte ont été faites à partir d'un broyat de rate de porc malade dilué à 10 p. 100 dans du CMFS (*) et centrifugé à $2.000 \mathrm{t} / \mathrm{min}$. pendant 10 minutes. Les souris ont été inoculées avec le surnageant. soit par voie intra-péritonéale à raison de $0,5 \mathrm{ml}$ par animal, soit par voie intra-cérébrale à raıson de $0,025 \mathrm{ml}$. Il n'a pas été observé de mortalité chez les souris inoculées après une durée 'd'observation de 3 semaınes à 1 mois. Passés ces délais, les animaux ont été sacrifiés, un examen des frottis de rate ou de cerveau colorés au May-Grünwald-Giesma n'a pas permis de frouver de rickettsies.

\section{b) Essai d'adaptation à l'œuf embryonné.}

Des œufs embryonnés de 5 à 6 jours ont été inoculés par la voie intra-vitelline avec un inoculum provenant d'un broyat de rate à partir d'un porc malade, sacrifié avant son décès. Le broyat a été dilué à 10 p. 100 dans du CMFS et centrifugé 10 minutes à $2.000 \mathrm{t} / \mathrm{m} ı n$. L'inoculation est faite à partir du surnageant. Les cufs morts à partir du $3 e$ jour suivant l'inoculation sont ouverts et les sacs vitellins prélevés et conservés au congélateur pour être inoculés à des ceufs neufs. II est également fait un frottis à partir des sacs vitellins. Cinq passages successifs ont été faits à partir de l'inoculum de rate : au cinquième passage, il n'a pas été possıble d'observer une mortalité importante sur les œufs inoculés, ni de trouver des ricketłsies dans les frottis de sac vitellin.

(*) Caicium magnesium foult salt solution.
30 Essai d'adaptation aux cultures de leucocytes.

\section{a) Matériel et méthode.}

Cultures de leucocytes de bovin : les leucocytes de bovin sont cultivés selon la méthode de TOKUDA et coll. (1962). Le sang est recueilli dans une éprouvette contenant $0,2 \mathrm{mg} / \mathrm{ml}$ d'héparine, puis aussitôt réparti dans des tubes à lamelle à raison de $2 \mathrm{ml}$ par tube. Ces derniers sont mis sur un portoir et inclinés à $40^{\circ}$ dans une étuve d̀ $37^{\circ} \mathrm{C}$. Au bout de 48 heures, les leucocytes se sont déposés et adhèrent à la surface du verre au niveau de la séparation des hématies et du plasma ; le sang est alors rejeté, les tubes sont rincés trois fois avec une solution de Hanks et le milieu suivant est introduit :

Sérum de bouf inactivé $\ldots \ldots \ldots \ldots 4$ parties Hanks LAYE .................. 1 partie

Les tubes sont alors mis à l'étuve à $37^{\circ} \mathrm{C}$ sur un portoir incliné à $40^{\circ}$. 48 heures après, les tubes sont vidés, le tapis cellulaire est inoculé ef on ajoute un milieu dont la composition est la suivante :

Sérum de bceuf inactivé.......... 1 partie Hanks LAYE $\ldots \ldots \ldots \ldots \ldots \ldots \ldots 4$ parties

Les tubes sont examinés quotıdiennement après l'inoculation.

Cultures de leucocytes de porc : les leucocytes de porc sont cultivés selon la technique légèrement modifiée de MALMQUIST et HAY (1960). Le sang est recueilli aux abattoirs dans une éprouvette contenant $0,2 \mathrm{mg}$ d'héparine par $\mathrm{ml}$. L'éprouvette est laissée sur la pallasse pendant 2 heures pour permettre la séparation des hématies ef du sérum. Les leucocytes sont prélevés par aspiration du sérum situé à la surface des globules rouges. II convient d'éviter de prélever des hématıes en trop grande quantité.

Le sérum ainsi prélevé est mélangé à 9 parties de milieu de culture. Celui-ci est constitué par un tiers de sérum normal de porc et deux tiers de milıeu 199 ou de EARLE LAYE. Ce mélange contenant de la pénicilline, de la streptomycine et de la mycostatine aux concentrations usuelles, est réparti en tubes, ces derniers sont placés en position inclinée stationnaire à l'étuve à $37^{\circ} \mathrm{C}$ Au bout de 24 heures, les leucocytes ont adhéré au verre et le milieu est élimıné. Les cellules sont inoculées avec $0,2 \mathrm{ml}$ de suspension viru- 
lente et après une incubation de 30 minutes à la température du laboratoire, un milieu neuf identique au précédent est introduit. Les tubes sont examinés chaque jour après l'inoculation.

Inoculum : il est constitué par un broyat de rate dilué à 5 p. 100 dans du CMFS contenant $100 \mathrm{UI}$ de pénicilline et 50 gamma.de streptomycine par millilitre et il est centrifugé 10 minutes d̀ $2.000 \mathrm{t} / \mathrm{min}$. Le surnageant est prélevé pour inoculation des tubes; chaque tube reçoit $0,2 \mathrm{ml}$ de liquide.

\section{b) Résultat.}

Cing passages successifs ont été faits sur leucocytes de porc et leucocytes de bovin. Les tubes sont laissés à l'étuve à $37^{\circ} \mathrm{C}$ entre 10 et 15 jours lors de chaque passage. If n'a pas été possible d'observer de lésions du tapıs cellulaire même après coloration. Un essai d'inoculation d̀ un veau fait à partir du cinquième passage sur leucocytes de bovin n'a pas donné de résultats.

\section{Nature de la rickettsie responsable.}

Cette rickettsie présente les caractères morphologiques du genre Erlichia (Rickettsia). Mais il ne s'agit pas de Rickettsia (Erlichia) suis, décrite par DONATIEN et GAYOT (1943) car cette espèce parasite à la fois les monocytes et les cellules des endathéliums vasculaires. Or, dans le cas présent, aucune rickettsie n'a été trouvée dans les celluies endothéliales.

L'existence de Rickettsia bovis dans la région et la présence dans la porcherie de veaux de race locale fait penser que cette dernière espèce pouvait être l'agent causal de la maladie.

Les expériences suivantes tendent à le démontrer :

a) Le sang d'un porc malade, inoculé par voie intraveineuse à un veau, déclenche chez lui un accès parasitaire avec présence de nombreuses rickettsies dans les monocytes. Les rickettsies apparaissent 7 jours après l'inoculation et persistent 5 jours, puis l'animal meurt sans avoir présenté de symptômes de rickettsiose

b) Un broyat de tiques ( 9 Hyalomma truncotum dont 1 of, 1 Rhipicephalus evertsi of et 1 Rhipicephalus musamae $\left.3^{3}\right)$ récoltées sur des veaux séjournant dans une stalle de la porcherie est inoculé à la fois d̀ un porcelet neuf et à un veau neuf. Des rickettsies sont identifiées 15 jours après l'inoculation chez le veau et 21 jours après chez le porc. Chez les deux animaux, elles persistent pendant deux mois durant lesquels interviennent des périodes d'éclipse pouvant durer jusqu'à 9 jours. Puis les rickettsies disparaissent sans que les deux animaux en aient souffert.

L'infection latente est donc possible chez le porc.

Bien que le manque d'animaux d'expérience n'ait pas permis de tenter la transmission directe du bœuf au porc, ni les essais de prémunition crolsée, les expériences précédentes permettent de conclure que la rickettsie observée chez le porc est Rickettsia bovis ou une espèce très voisine commune au porc et aux bovidés.

\section{Mode de transmission.}

En présence d'une rickettsiose, les tiques doivent être recherchées ; mais aucun des animaux de cet élevage (malades ou en bonne santé) n'est trouvé porteur de ces ectoparasites. Cette rickettsıose dolt donc être considérée comme une maladie de sortie provequée par un stress quelconque.

II est d'ailleurs remarquable de constater que l'apparition des cas cliniques coincide toujours avec de brusques balsses de la température ambiante. Un nombre variable de porcs présente alors les premiers symptômes de la maladie qui évolue ensuite vers la mort. D'autre part, l'inoculation de broyat de tiques mentionnée plus haut a montré que l'infection inapparente existalt chez le porc.

Afin de confirmer cette hypothèse, un jeune pore de race locale pris au hasard dans la porcherie, reçoit dans le péritoine $5 \mathrm{ml}$ d'hydroxyde d'alumine (gel d̀ 1 p. 100 de matière sèche) pour provoquer un choc artificiel. Quatre jours plus tard, la température atteint $40^{\circ} \mathrm{C}$ et les symptômes de rickettsiose apparassent. Le porc meurt après une évolution de 3 jours. Les lésions sont celles déjà observées et les rickettsies, mises en évidence dans les étalements de sang au cours de la maladie, sont ensuite retrouvées dans les frottis d'organes.

Un broyat de la rate de cet animal, inoculé d̀ un autre porc d'expérience provoque en quelques jours une rickettsiose mortelle.

Cette épidémıologie ne peut cependant être invoquée pour quinze animaux importés de France un mois avant l'apparition de la maladie 
et apparemment morts de rickettsiose (*). II faut supposer que ces animaux sont morts des suites d'une infection primaire.

\section{CONCLUSION}

Le mode d'apparition de la maladie, les expériences de prémunition par inoculation au porc d'un broyat de tiques et l'apparition de la rickettsiose après un choc expérimental permettent de conclure que chez les porcs de race locale, il s'agit d'une maladie de sortie chez des

(*) Deux de ces animaux seulement ont été adressés au laboratoire à des fins d'analyse. animaux en état d'ınfection latente. Chez les porcs importés, seule l'infection primaire grave peut être invoquée car l'existence d'une rickettsiose porcine n'a pas été signalée en France.

La rickettsie responsable présente tous les caractères du genre Erlichia. II s'agit solt d'Erlichia bovis accasionnellement pathogène pour le porc, solt d'une rickettsie très voisıne commune aux suidés et aux bovidés.

Institut d'Elevoge el de Médecine Vétérinaire des Poys tropicaux, Maisons-Alfort. Laborotoire national de l'Elevage et de Recherches vétérinarres. Dakar-Honn.

\section{SUMMARY}

\section{Rickettsiosis of monocytes in pig in Senegal}

An enzootic outbreak was reported during the cool season in a pig farm near Dakar and was shown to be caused by a Rickettsia. The symptoms, the evolution and the micro and macroscopic lesions have been described. Only Aureomycin was effective in the treatment, if used in the first stage of the disease. The Rickettsia evidenced, was not Rickettsio suis, but as shown by experiments, either Rickettsia bovis, or another similar species common to pig and cattle. The causative agent could not be cultivated. This Ricketssosis seemed to occur following a stress in latent infected animals.

\section{RESUMEN}

Rickettsiosis de los monocitos observada en el cerdo en Senegai

Se observa una enzootia identıficada como una ricketısiosis en una explotación del cerdo de los alrededores de Dakar. Se describen los síntomas, la evolución y las lesiones micro y macroscopicas. Desde el punto de vista del tratamiento, sola es activa la aureomicina con tal de que sea utilizada precozmente. No es Rickettsı suis la rickettsı observada pero, como lo demuestran las experimentaciones, sea Rickeftsia bovis, ya sea una especie vecina, encontrada en los cerdos y en los bovinos. No se pudo cultivar el germen. Esta rickettsiosis aparece en animales precavidos al momento de un choque fisiológico.

\section{BIBLIOGRAPHIE}

DONATIEN (A.) et GAYOT (G.). - Rickettsiose générale du porc. Arch. Inst. Pasteur Alger, 1943, 21, (1) : 5.

MALMQUIST ef HAY, - Hemadsorption and cytopathic effect produced by PPA virus in swine bone marrow and buffy coat culture. Amer. J. vet. res., 1960, 21, 104-108.

RIOCHE (M.). - Lésions microscopiques de la rickettsiose générale bovine à Rıckettsia
(Erlichia) bovis (Donatien et Lestoquard, 1936). Rev. Elev. Méd. vét. Pays trop., 1967, 20, (3) : 415-27.

TOKUDA (G.), FUKUSHO (K.), MORIMOTO (T.) and WATANABE (M.). - Studies on rinderpest virus in bovine leucocyte culture.

1. Cultivation of leukocytes and appearance of inclusions in infected cells. Nat. Inst. anim. Hith. Q. Tokyo, 1962, 2, 189-200. 